\title{
Nuevos modelos de soberanía. Kosovo y la soberanía irrelevante
}

Ana M. Jara Gómez

Investigadora de la Universidad de Jaén

Correo Electrónico: ajara6@ugr.es

Resumen: La cuestión de la soberanía, en permanente evolución, tiene una enorme importancia tanto en el ámbito jurídico y académico, como en el mundo de la Realpolitik. En el caso de Kosovo es posible concluir que la cuestión de la soberanía resulta de menor relevancia que la gobernanza, dado que, al menos durante el periodo 1999-2008, ningún Estado puede ejercerla efectivamente ni reclamar los atributos y competencias que a ella acompañan. Tras la intervención militar de la OTAN, Naciones Unidas desplegó una administración territorial que colocaba todos los atributos de la soberanía y los instrumentos de poder en manos de agentes extranjeros cambiantes, no vinculados al territorio o a la sociedad de Kosovo. La autoridad del Estado es reemplazada y superada por la autoridad funcional de una institución internacional que administra y gobierna el territorio.

Palabras clave: Soberanía, Kosovo, UNMIK, condicionalidad, estándares.

Abstract: The question of sovereignty, though constantly evolving, has an enormous relevance both in the legal and the academic fields, and in the world of the Realpolitik. In the case of Kosovo it is possible to conclude that the question of sovereignty turns of less relevance than governance given that at least between 1999 and 2008 there is no state that can actually neither exercise it nor assert the competences and attributes attached to it. After the military intervention of NATO, United Nations deployed territorial administration which placed all the attributes of sovereignty and the instruments of power in the hands of foreign changing agents, unrelated to the territory and to the society of Kosovo. State authority is replaced and superseded by the functional authority of an international body that manages and governs the territory.

Keywords: sovereignty, Kosovo, UNMIK, conditionality, standards. 
La concepción clásica de la soberanía aparece intrínsecamente ligada a la idea de Estado, asumiendo que la soberanía es un atributo esencial e indubitable de los Estados. De este modo se puede concluir que un Estado o es soberano o no es propiamente un Estado.

Sin embargo, lo que resulta sencillo concluir desde el relato académico y la perspectiva doctrinal, no lo es tanto cuando de hechos y concretas situaciones históricas se trata: la soberanía está pues sujeta a interpretaciones y lecturas muy variadas. Ningún Estado posee la total y plena capacidad de disposición sobre todos y cada uno de espacios en los que se despliegan la vida y las acciones de los individuos a él sometidos. Ninguno detenta, por tanto, propiamente el poder absoluto sobre todos los campos de acción de los asociados ni sobre todos los eventos que tienen lugar en el ámbito de su jurisdicción.

No obstante, el hecho fundamental es que, reclamando supremacía, el soberano no está en posición de desplazar la responsabilidad por sus acciones a otro espacio político, sujeto o agente político-institucional, etc. Casi todos los Estados de hoy, democráticos o autoritarios, se basan en la idea de soberanía popular, la idea de que el Estado existe para cumplir la voluntad del pueblo. Si el pueblo es formalmente soberano, cualquiera dentro del Estado es, en última instancia, responsable del devenir de los acontecimientos sociales. El Estado proporciona a la sociedad una fuente de poder estable que hace posible considerar a un individuo o grupo responsable de ciertas decisiones políticas.

En 1999, el Consejo de Seguridad de la ONU autorizó una presencia internacional en Kosovo explícitamente bajo el capítulo VII de la Carta de Naciones Unidas, que ofrece cobertura legal suficiente para el despliegue de una administración territorial, que se habría de llamar UNMIK, y que asumiría las funciones gubernamentales y el uso de la fuerza. No se trata solo, por tanto, de que la Resolución del Consejo de Seguridad 1244 dispusiera el marco para la implementación del acuerdo de $\mathrm{paz}^{1}$, sino que el estatus constitucional de Kosovo desde junio de 1999 a febrero de 2008 descansa en ella.

\footnotetext{
${ }^{1}$ Resolución del Consejo de Seguridad 1244, de 10 de junio de 1999, disponible en http://daccess-ddsny.un.org/doc/UNDOC/GEN/N99/172/89/PDF/N9917289.pdf?OpenElement.
} 
Un asunto de tanta relevancia, lugar común en la agenda de la filosofía política contemporánea, merece ser analizado en el nuevo marco de las relaciones internacionales, tal y como se viene perfilando en la primera década de este siglo. Sentado, pues, que la soberanía, aunque en permanente evolución, tiene una enorme importancia en el ámbito jurídico y dogmático, y, como ha quedado dicho en el mundo de la realidad sociopolítica. En las páginas siguientes nos proponemos confirmar que en el caso de Kosovo la soberanía resulta prácticamente irrelevante dado que, al menos durante el periodo 1999-2008, ningún Estado puede ejercerla efectivamente ni reclamar las competencias que a ella acompañan.

\section{1.- La Resolución 1244 y la cuestión de la soberanía}

Los impulsos políticos para el repliegue tras las fronteras fortificadas en alianza con la aplicación de políticas de contención, están en conflicto con las políticas internacionales de intervencionismo selectivo y con el establecimiento de 'protectorados'. Este conflicto queda evidenciado en respuestas como la que se dio al conflicto de Kosovo, que fulminó simultáneamente la nonata soberanía tradicional de Serbia $^{2}$ y las aspiraciones de autodeterminación inmediata de Kosovo. Se hace

\footnotetext{
2 Ó TUATHAIL, G., "The Postmodern Geopolitical Condition: States, Statecraft, and Security at the Millennium", Annals of the Association of American Geographers, vol. 90, no 1, marzo de 2000, p. 175. No deseo dejar sin aclaración el hecho de que la soberanía de Serbia sobre Kosovo, en términos jurídicos, no llegó a nacer. Siguiendo los argumentos del historiador Noel Malcolm, hay que tener en cuenta la diferencia entre ocupación y efectiva posesión de un territorio. Serbia ocupó la mayor parte de Kosovo entre noviembre de 1912 y noviembre de 1915, pero Kosovo no era legalmente parte de Serbia. Es doblemente cierto que la ocupación serbia no puede asemejarse a una incorporación legal de Kosovo a Serbia: es cierto en los términos de la propia legislación constitucional de Serbia, y es cierto también en términos de Derecho Internacional. La Constitución serbia de 1903, que estaba en vigor en 1912, decretaba que no podían alterarse las fronteras de Serbia sin el acuerdo de una especial y ampliada 'Gran Asamblea Nacional'. Tal Asamblea no fue nunca reunida para ratificar la anexión de Kosovo. En el Derecho Internacional, el territorio de un Estado conquistado por otro se convierte legalmente en parte del Estado vencedor cuando la transferencia es acordada formalmente por ambos contendientes en un Tratado tras la guerra. Dos de estos Tratados fueron redactados (el Tratado de Londres en 1913 y el Tratado de Estambul en 1914), pero ninguno fue ratificado por Serbia. Por tanto, en términos estrictamente jurídicos, Kosovo era parte de Serbia entre 1912 y 1915 tanto como lo era de AustriaHungría, Alemania o Bulgaria (los siguientes Estados ocupantes) entre 1915 y 1918. Es también Ilamativo que el gobierno serbio no aprobara nunca una legislación que hiciera a los albaneses de Kosovo ciudadanos de Serbia; éstos ganaron su nueva ciudadanía cuando ésta les fue concedida por el Estado yugoslavo (el Reino de Serbios, Croatas y Eslovenos) en 1928. En ese momento se podía ya invocar un
} 
necesario distinguir entre la soberanía formal, tradicional de la República Federal de Yugoslavia, que es la que está en juego cuando la OTAN lleva a cabo su intervención militar, y la soberanía sobre Kosovo, que cobra el protagonismo cuando termina la intervención.

Los ataques serbios a civiles de Kosovo en la década de los noventa evidenciaban atrocidades a gran escala y propiciaron una actuación militar de la OTAN contra la que fuera República Federal de Yugoslavia ${ }^{3}$. Ello dio lugar a la puesta en marcha por parte de la ONU del programa de reconstrucción más ambicioso de su historia. La OTAN comenzó sus ataques aéreos el 24 de marzo de 1999 y el día 9 de junio de 1999, los jefes de las fuerzas militares serbias y de la OTAN llegaron finalmente a un Acuerdo Militar Técnico para el abandono de Kosovo por parte de las tropas serbias ${ }^{4}$. Al día siguiente, la OTAN suspendió los bombardeos y acordó con Rusia una Resolución del Consejo de Seguridad que preveía una presencia internacional de seguridad militar y otra civil en Kosovo bajo el patrocinio de las Naciones Unidas. Ambas 'presencias' constituyen UNMIK, la misión de Naciones Unidas en Kosovo.

Cuando se desplegó UNMIK, su máximo responsable, el Representante Especial del Secretario General, publicó una ley decretando que "toda la autoridad legislativa y ejecutiva en Kosovo, incluyendo la administración de justicia, está conferida a UNMIK y es ejercida por el Representante Especial del Secretario

\footnotetext{
criterio de Derecho Internacional más pragmático (control de largo plazo y administración establecida con reconocimiento de otros Estados) para argumentar que Kosovo no podía ser considerado como legalmente parte del Estado otomano o turco. Pero la conclusión es inevitable en todo caso: mientras Kosovo se convirtió en algún momento en parte del Estado yugoslavo, no fue parte del Estado serbio en el periodo entre 1912 y 1918. Por tanto Kosovo se incorporó a Yugoslavia, no a Serbia. MALCOLM, N., "Kosovo and Bosnia: three points", Bosnian Report, Marzo - Mayo 1998, New Series no 3. El artículo está disponible en http://www.bosnia.org.uk/bosrep/marmay98/kosovo.cfm

${ }^{3}$ Sucesora de la República Federal Socialista de Yugoslavia. Usaremos el término para referirnos a Serbia y Montenegro hasta el año 2003, año en que cambiaron su nombre. Serbia pasará a llamarse simplemente Serbia a partir de 2006, momento en que se disuelve la Unión de Estados de Serbia y Montenegro.

${ }^{4}$ Véase Military Technical Agreement between the International Security Force (KFOR) and the Governments of the Federal Republic of Yugoslavia and the Republic of Serbia, disponible en: http://www.nato.int/kfor/kfor/ documents/mta.htm. Se llama comúnmente 'Acuerdo de Kumanovo'.
} 
General" "Estas tres líneas no son otra cosa que el diseño de una soberanía absoluta sobre el territorio de Kosovo, sin cortapisas ni mecanismos de control o responsabilidad. Es esta una soberanía que coloca todos los instrumentos de poder en manos de agentes extranjeros cambiantes, no vinculados al territorio o a la sociedad y cuyas prioridades están sujetas a intereses y procesos decisorios que se encuentran a miles de kilómetros de Kosovo ${ }^{6}$.

La Resolución 1244 afirma también en su preámbulo "el compromiso de todos los Estados Miembros con la soberanía y la integridad territorial de la República Federal de Yugoslavia”. Establece también que la administración provisional busca el establecimiento para Kosovo de "una autonomía sustancial dentro de la República Federal de Yugoslavia”. Se ha llegado a calificar a estas dos líneas como "una visión esquizofrénica de la soberanía del Estado"7, afirmación que no estamos en condiciones de rebatir, sobre todo porque a esta visión ha de añadirse la amplitud de la autoridad de UNMIK, que roza un modelo cercano al absolutismo. Las actuaciones de UNMIK no se encaminaron a construir la soberanía de Kosovo para el propio Kosovo, es decir, a su posible secesión o independencia. El dilema

\footnotetext{
${ }^{5}$ Ley UNMIK 1999/01 "On the Authority of the Interim Administration in Kosovo", de 25 de julio de 1999.

${ }^{6}$ Resulta de la mayor utilidad adaptar a este contexto una descripción muy acertada que realiza el profesor David Kennedy de la gobernanza global y que, a pesar de estar plagada de interrogantes, es sumamente esclarecedora: "La idea de que la entidad es un grupo de capacidades, permisos y prohibiciones fragmentado y fluido es muy familiar en nuestro orden económico. También lo es nuestro orden político. $\mathrm{Y}$ es algo cuya estructura puede ser estudiada y trazada y donde la gente de Chevron decide si paga impuestos en Nigeria o si colocan todas sus pérdidas en Nigeria de modo que no tengan que pagar impuestos. Esta clase de decisiones, me parece, se centran en momentos de gobernanza global. De modo que las personas que gobiernan, me parece, son expertos en todas estas localizaciones. Podemos preguntarles qué están haciendo. Podemos preguntarles por qué creyeron que era una buena idea. Y podemos estudiar el proceso a través del cual desplazan la responsabilidad de uno a otro de modo que todos están convencidos de que no surge en ellos. Es esta una sociología muy útil del proceso actual de toma de decisiones. Así que a la pregunta de cómo yo trazaría el mapa, lo haría así, yendo a los lugares donde la gente decide cosas que tienen consecuencias para otra gente, y preguntándoles por qué pensaron que tenían el poder de hacerlo. Por qué pensaron que esa decisión era correcta. Y por qué no han experimentado que son éticamente responsables de las consecuencias". KENNEDY, D., "How Should Sovereignty be Defended?", en GOUREVITC, A., (ed.), Politics Without Sovereignty: A Critique of Contemporary International Relations, UCL Press, Londres, 2007, p. 199.

${ }^{7}$ CHINKIN, C. M., "Kosovo: A "Good" or "Bad" War?", The American Journal of International Law, vol. 93, no 4, octubre de 1999, p. 845.
} 
entre la integridad territorial de Serbia y la autodeterminación de Kosovo no se resolvió ni figuró en las agendas durante los años en que UNMIK gozó de los plenos poderes del Estado, y este hecho relevante determina que su trayectoria esté marcada por los apuros que implica la ausencia de destino y la no cooperación entre albaneses y serbios de Kosovo.

En este sentido la Resolución tiene el efecto de crear una situación híbrida que separa la soberanía de jure del ejercicio de facto de los poderes públicos ${ }^{8}$. Tal vez estos hechos muestran que una de las claves para el siglo XXI es lograr un compromiso entre los principios de autodeterminación y la santidad de las fronteras, tema que, por razones obvias, es imposible abordar en este artículo 9 .

UNMIK tomó numerosas decisiones que desmienten la intención de un respeto real hacia cualquier otra soberanía sobre Kosovo ajena a ella misma: estableció un sistema de aduanas completamente independiente de la República Federal de Yugoslavia (formada entonces por Serbia y Montenegro), expidió pasaportes y documentos de identidad a los kosovares e introdujo una nueva moneda de curso legal ${ }^{10}$. Todas estas decisiones se encuentran en el núcleo de la soberanía y reflejan una interpretación muy amplia de los poderes concedidos en la Resolución 1244, prácticamente irreconciliable con la soberanía de la República Federal de Yugoslavia que la propia Resolución decía garantizar ${ }^{11}$.

En su informe de 12 de junio de 1999, el Secretario General de la ONU presentó la e structura operativa y los conceptos básicos para la puesta en marcha de la Misión de Administración Provisional de las Naciones Unidas en Kosovo,

\footnotetext{
${ }^{8}$ FRIEDRICH, J., "UNMIK in Kosovo: Struggling with Uncertainty", Max Planck Yearbook of United Nations Law, vol. 9, no 1, 2005, p. 142.

9 SCHARF, M. P., "Earned Sovereignty: Juridical Underpinnings", Denver Journal of International Law and Policy, vol. 31, 2004, p. 373.

${ }^{10}$ Ley UNMIK 1999/4, “On the Currency permitted to be used in Kosovo”, de 2 de septiembre de 1999.

11 En el documento llamado Memorandum of the government of the RFY on the implementation of United Nations Security Council Resolution 1244 (1999), publicado en Belgrado el 3 de noviembre de 1999, la República Federal de Yugoslavia enumera una larga lista de protestas contra las violaciones a su soberanía.
} 
UNMIK $^{12}$. Se pretendía lograr un formato que permitiese el desarrollo integrado de las innumerables actividades de la comunidad civil internacional en Kosovo bajo una cadena de mando única, precisa y jerárquica ${ }^{13}$. La presencia internacional militar se materializó en KFOR (siglas para Kosovo Force). Autorizada por la Resolución en paralelo a la presencia civil, era independiente de ésta, si bien ambas habían de coordinarse y apoyarse sin que hubiera jerarquía entre ellas. La tarea primordial de KFOR consistía en evitar el surgimiento de nuevas hostilidades manteniendo el alto el fuego, y vigilar la retirada permanente de las fuerzas yugoslavas y serbias al territorio kosovar ${ }^{14}$.

UNMIK fue establecida en un principio con un diseño, unas funciones y unos objetivos que han sido extraordinariamente cambiantes con el paso del tiempo y con la evolución de los acontecimientos. Sin duda un punto de inflexión ha sido la unilateralmente proclamada independencia de Kosovo, que ha marcado de algún modo el principio de su fin. La misión nació compuesta por cuatro Pilares, cada

\footnotetext{
${ }^{12}$ Informe del Secretario General presentado de conformidad con el párrafo 10 de la Resolución 1244 (1999) del Consejo de Seguridad, S/1999/672, de 12 de junio de 1999. Todos los Informes del Secretario General referentes a Kosovo están disponibles, en castellano, en el enlace http://www.un.org/es/peacekeeping/missions/unmik/reports.shtml.

${ }^{13}$ Alfonso J. Iglesias Velasco, “La Misión de Administración Provisional de las Naciones Unidas en Kosovo
(UNMIK)", Revista CIDOB d’Afers Internacionals, №. 56, 2001-2002, p. 121.

${ }^{14}$ KFOR debía también desmilitarizar la guerrilla del UÇK y garantizar un entorno seguro que hiciera posible el regreso de los refugiados y desplazados internos, mantener el orden y la seguridad hasta que se desplegara la presencia civil internacional, supervisar la remoción de minas, y acometer la vigilancia de las fronteras. Este parece un mandato muy amplio pero necesario para un lugar donde no quedaba policía local, sistema legal, infraestructuras de obras públicas, u organización institucional o social consolidada. En principio KFOR estaba compuesta por unos 50,000 hombres y mujeres de los países miembros de la OTAN, de los socios y de algunos no miembros (Rusia), bajo un mando único. Se redujo en 2002 hasta unos 39,000 efectivos y debido al incremento de la situación de seguridad la presencia fue menguando hasta unos 17.500 en junio de 2003. Los ministros de exteriores de la OTAN, acordaron en diciembre de 2008, cuando Kosovo ya había declarado su independencia, que la presencia de KFOR se quedaría en el país sobre la base de la Resolución 1244. En 2009 los ministros de defensa decidieron ir ajustando las fuerzas KFOR a la evolución presente, reduciendo el número de fuerzas para mantener lo que llamaron 'presencia disuasoria' de modo que para el inicio de 2010 el número de hombres había disminuido hasta quedar unos 10,200. Posteriormente el Consejo del Atlántico Norte autorizó el repliegue de la mitad de la fuerza en el territorio, quedando por tanto unos 5.000 hombres de KFOR en Kosovo. Véase www.nato.int/kfor/.
} 
uno de los cuales, bajo el paraguas de la ONU, tenía un objetivo y un campo de acción propio:

a) El primer Pilar, de asistencia humanitaria, estaba dirigido desde el Alto Comisionado de Naciones Unidas para los Refugiados (ACNUR-UNHCR);

b) El segundo Pilar, responsabilidad directa de Naciones Unidas, es el de la administración civil, que incluyó al principio los componentes de policía y administración de justicia;

c) El tercer Pilar, de democratización y fortalecimiento institucional, es (todavía) responsabilidad de la Organización para la Seguridad y Cooperación en Europa (OSCE); y

d) El cuarto componente de UNMIK, el Pilar de reconstrucción y desarrollo económico, fue encargado a la Unión Europea y financiado principalmente por la Comisión, y desapareció el 30 de junio de 2008.

La principal tarea de UNMIK consistía, pues, en promover el establecimiento de un régimen de autogobierno en Kosovo, teniendo en cuenta los Acuerdos de Rambouillet, desempeñar las funciones administrativas civiles esenciales y organizar y supervisar el desarrollo de instituciones democráticas de autogobierno durante un período transitorio ${ }^{15}$. Asimismo, tenía encargada la tarea de facilitar el proceso político para consensuar el estatus jurídico definitivo de Kosovo, apoyar la reconstrucción de la infraestructura y el auxilio humanitario, mantener la ley y el orden público mediante el despliegue de agentes internacionales de policía, promover el respeto de los derechos humanos, y garantizar el retorno seguro y libre a sus hogares de los refugiados y de las personas desplazadas.

Queda patente que la ausencia de un sistema normativo estatal no necesariamente refleja un territorio ingobernado, sino un territorio gobernado mediante formas alternativas a las estatalistas o a las propias del modelo regulativo estatal. En la actualidad existen muchos territorios en que la autoridad y el poder se comparten entre actores de formas dinámicas no necesariamente estadocéntricas o

\footnotetext{
15 Antes del ataque de la OTAN, a través del llamado "Grupo de Contacto" -formado por EEUU, la Federación Rusa, Alemania, Francia, Italia y el Reino Unido se había intentado sin éxito negociar un acuerdo entre Belgrado y los kosovo albaneses en el castillo francés de Rambouillet.
} 
informadas por estrategias de control claramente articuladas y unificadas. En estos casos, la visión de régimen gobernante que se sugiere es "aquélla que implícitamente incluye los efectos condicionantes espaciales de la historia y reconoce que la soberanía es objeto de negociación constante a través de la interacción de actores con muy diversas intenciones, recursos y estrategias"16. Quizá una respuesta válida a la pregunta de en qué consiste la soberanía debería buscarse desde dentro de un contexto nacional y político concreto, y en el carácter de un Estado y una sociedad específicos.

A todo lo dicho hasta el momento debemos añadir otra cuestión controvertida, determinar qué significa ser soberano en las relaciones internacionales modernas. Se trata de averiguar cuáles son los poderes, privilegios, derechos y obligaciones que confiere el estatus de soberanía, más específicamente, saber si la soberanía es una idea jurídica o política, si los Estados en virtud de su estatus de soberanos gozan de unos derechos y obligaciones idénticos. O bien, si la soberanía confiere diferentes derechos e impone distintas obligaciones, según se trate de potencias mundiales o pequeños Estados. En definitiva, la soberanía puede contemplarse como absoluta, algo que puede perderse o ganarse, o como algo variable que puede aumentar o disminuir según los $\operatorname{casos}^{17}$.

La primera visión, en la que la soberanía sería un 'monolito', se identifica con el concepto westfaliano y con las palabras de Samuel Johnson: "En la soberanía no hay gradaciones. Puede haber realeza limitada, puede haber consulado limitado; pero no puede haber gobierno limitado. En todas las sociedades debe existir un poder u otro desde el que no hay apelación, que no admite restricciones, que impregna el todo de la comunidad, regula y ajusta toda la subordinación, que promulga leyes y las deroga, que levanta y anula judicaturas, extiende o restringe privilegios, elude el cuestionamiento y el control y está limitado exclusivamente por la necesidad

\footnotetext{
${ }^{16}$ CLUNAN, A. L., y TRINKUNAS, H. A., Ungoverned Spaces: Alternatives to State Authority in an Era of Softened Sovereignty, Stanford University Press, 2010, p. 168.

17 POTTER, D. W., "State Responsibility, Sovereignty, and Failed States", Trabajo presentado a la Australasian Political Studies Association Conference, Universidad de Adelaida, 29 septiembre- 1 de octubre de 2004, pp. 9 y $\quad$ ss. Disponible en https://www.adelaide.edu.au/apsa/docs_papers/Others/potter.pdf.
} 
física" ${ }^{18}$. Desde esta perspectiva, un Estado soberano disfruta de todos los privilegios de la soberanía simultáneamente, tiene población, gobierno y territorio, es supremo internamente, reconocido internacionalmente, etc., y la soberanía es indivisible, o se tiene por completo o no se tiene y todo el que la tenga, por lo tanto, independientemente de su tamaño, riqueza o poder militar, se beneficiará de los mismos privilegios legales y tendrá los mismos derechos y deberes que los demás Estados coetáneos.

Desde la segunda visión, la soberanía podría verse como una 'cesta', cuyo contenido varía de Estado a Estado. Los atributos de la soberanía serán variables y también sus derechos y deberes correspondientes. En este modelo la soberanía existe en grados y mientras algunos Estados poderosos pueden disfrutar de absoluta soberanía, otros encontrarán la suya cambiante, en continuo desarrollo o truncada. Esta concepción de la soberanía, aplicada a territorios concretos, posibilita el examen empírico de la 'cesta' de atributos soberanos de cada Estado para poder determinar el alcance de sus derechos y obligaciones y se acepta, generalmente, que hay Estados que son más soberanos que otros ${ }^{19}$. David Chandler advierte de los peligros de 'desgajar' la soberanía, afirmando que algunos autores superan los límites del deber académico en sus intentos de asegurarnos que los Estados no occidentales se encuentran sanos y salvos y mantienen responsabilidades soberanas $^{20}$. Explica que mientras estos teóricos se alegran de abandonar el contenido de la soberanía del Estado, la capacidad de autogobierno, buscan resucitar la forma de soberanía del Estado como un cascarón vacío. En efecto, la cáscara es despojada de su contenido. Una vez 'desgajada' (unbundled) la soberanía, de modo

\footnotetext{
18 JOHNSON, S., Taxation no Tyranny. An Answer to the Resolutions and Address of the American Congress, publicado por T. Cadell en Londres, 1775. La edición del texto correspondiente a la publicación de Pafraets \& Company, New York, 1913, The Works of Samuel Johnson, vol. 14, pp. 93-144, está disponible en: http://www.samueljohnson.com/tnt.html.

${ }^{19}$ POTTER, D. W., State Responsibility..., op. cit. pp. 9 y ss.

${ }^{20}$ El autor se refiere a los teóricos Stephen Krasner y Robert Keohane. Véanse, por ejemplo, KRASNER, S. D., Sovereignty. Organized Hypocrisy, Princeton University Press, 1999; y del mismo autor, "Rethinking the Sovereign State Model", Review of International Studies (2001), 27, pp. 17-42, y KEOHANE, R. O., "Political Authority after Intervention: Gradations in Sovereignty", en HOLZGREFE, J. L., y KEOHANE, R. O., Humanitarian Intervention. Ethical, Legal, and Political Dilemmas, Cambridge University Press, 2003, pp. 275-298.
} 
que el ideal clásico de soberanía westfaliana se abandone incluso como aspiración, tanto los Estados como la soberanía deben ser defendidos. De este modo, este ‘abandono' o ‘desgajamiento' no debe ser reconocido públicamente, y no significa que el Estado deba ser abandonado o la soberanía desacreditada como concepto ${ }^{21}$.

\section{2.- La dispersión de la soberanía kosovar}

En Kosovo, curiosamente, podemos encontrar ambas soberanías, la absoluta, en términos de Johnson, y otra 'relativa', variable y gradual. La soberanía absoluta queda atribuida a UNMIK por sus propias leyes, que le conceden virtualmente todas las competencias que pueden ejercerse en un territorio. La soberanía local representa aquella gradual, que adquiere competencias poco a poco, estando siempre sometida a una mayor autoridad. Además de la gradación horizontal o 'tradicional' de la soberanía, existe una especie de gradación vertical que afecta a la soberanía absoluta que se ejerce en Kosovo. Dado que UNMIK es un órgano creado por y dependiente de Naciones Unidas, los Estados miembros de la ONU ejercen una suerte de 'soberanía de segundo grado', y dirigen y orientan las actuaciones del soberano directo $^{22}$. Pero esto no es todo. También se da en Kosovo un tipo de soberanía 'dual', de muy difícil conceptualización, que tiene lugar en Mitrovicë/Mitrovica, al norte del país, donde las instituciones kosovares de nueva creación carecen de representación significativa y UNMIK no logra tampoco el control político, que se podría decir está en manos de Serbia pero separado de ella ${ }^{23}$. No cabe duda de que

\footnotetext{
${ }^{21}$ CHANDLER, D., "How State Building Makes States Weak", Spiked, 24 de octubre de 2005. Disponible en http://www.spiked-online.com/index.php/site/article/515/.

${ }^{22}$ Esta es una reflexión que debo agradecer al profesor de Filosofía del Derecho de la Universidad de Granada, D. Manuel Escamilla.

${ }^{23}$ En una consulta popular celebrada el 14 y el 15 de febrero de 2012, declarada ilegal por el gobierno de Kosovo, Belgrado y UNMIK, el 99,5\% de los serbios kosovares rechazó la autoridad del gobierno kosovar de mayoría albanesa de Prishtinë/Priština, según los resultados preliminares del recuento de votos. La consulta tuvo lugar en las ciudades de Mitrovicë/Mitrovica, Zvečan/ Zveçan Zubin Potok y Leposavić/Leposaviq. RIA Novosti, Belgrado, 16 de febrero de 2012, http://sp.ria.ru/international/20120216/152731696.html. Véase también International Crisis Group, North Kosovo: Dual Sovereignty in Practice, Europe report no 211, 14 de marzo de 2011, disponible en http://www.crisisgroup.org/en/regions/europe/balkans/kosovo/211-north-kosovo-dual-sovereignty-in-
} 
Kosovo es un caso único, su soberanía es colectiva, divisible y condicionada, ganada y compartida, suspendida y en constante proceso de evolución y evaluación ${ }^{24}$.

Es de gran utilidad tener presentes las implicaciones generales del fenómeno que algunos llaman internacionalización del territorio de Kosovo. Pero en el fondo, esto no deja de ser la simplificación o la trivialización de una situación mucho más grave y profunda, que podemos calificar de ausencia de contrato social. Parece ser que un territorio 'internacionalizado' es un territorio libre de soberanía, o más bien, un territorio en que se produce una disociación entre soberanía y jurisdicción. Los territorios internacionalizados se situarían entre dos poseedores de poder público: la jurisdicción de una administración internacional y la soberanía del Estado territorial. Los poderes de gobierno se emplazan en las autoridades internacionales con jurisdicción mientras el soberano territorial mantiene el título formal sobre la entidad gobernada. Esta disociación, opinan algunos autores, se produce típicamente en casos en que la autoridad del Estado es reemplazada y superada por la autoridad funcional de una institución internacional que administra y gobierna el territorio, bien exclusivamente, bien en cooperación con las autoridades locales del lugar ${ }^{25}$.

En estos casos, en los que existe una autoridad funcional resulta necesario usar criterios de eficiencia, optimizar, calcular tal vez un número de pérdidas, o de víctimas. Cuando lo social es fluido, un concepto social del derecho debe ser también fluido, un derecho pragmático. Todo debe tornarse negociable, revisable,

practice.aspx. Están por ver los resultados de un reciente acuerdo entre los Estados de Serbia y Kosovo, posible al ser establecida, como condición para obtener el estatus de candidato a la adhesión a la Unión Europea, la normalización de las relaciones entre ambos países. Véase, por ejemplo, BARLOVAC, B., "Kosovo and Serbia Reach Historic Deal in Brussels", Balkan Insight, 19 de abril de 2013.

24 Un ejemplo de los procesos evaluadores es la decisión tomada el 1 de julio de 2012 por el International Steering Group for Kosovo. El grupo decidió conceder soberanía nacional plena a Kosovo a partir de septiembre de 2012, decretando el final de la supervisión internacional. El Grupo, compuesto por 23 países europeos, Turquía y los Estados Unidos ha supervisado la independencia desde que se produjo, contribuyendo al establecimiento de instituciones democráticas. Peter Feith, el jefe de la International Civilian Office del Grupo en Kosovo manifestó en Viena que Kosovo se ha convertido en un país moderno, democrático y multiétnico aunque queda mucho por hacer, pero destaca su "notable progreso" en los últimos años. Radio Free Europe 02-07-2012, disponible en http://www.rferl.org/content/international-monitors-grant-kosovo-sovereignty/24632544.html.

${ }^{25}$ STAHN, C., The Law and Practice of International Territorial Administration. Versailles to Iraq and Beyond, Cambridge University Press, 2008, p. 541. 
para lograr el resultado deseado ${ }^{26}$. Uno de los inconvenientes de estas normas pragmáticas sociales no estrictamente jurídicas es la dificultad de identificar a la sociedad. La norma jurídica permite identificar a un legislador como fuente y someterle a control, pedirle cuentas, realizarle preguntas y solicitarle enmiendas. La norma social es creada por la sociedad con el consiguiente problema de qué pueda entenderse por sociedad. Por ejemplo, serían tal vez 'sociedad' los medios de comunicación, que pueden legitimar un conflicto armado y deslegitimar otro sobre criterios poco o nada $\operatorname{claros}^{27}$, igual ocurre si se incluye a las instituciones económicas transnacionales o a las empresas multinacionales en este concepto de 'sociedad'.

En Kosovo, la comunidad internacional se centró principalmente en la construcción de instituciones, por considerarlas un componente esencial de la paz duradera y sostenible. El 15 de mayo de 2001, se publicó la ley UNMIK llamada Marco Constitucional para el Autogobierno Provisional en Kosovo con la intención de proporcionar la base para la formación de instituciones locales

\footnotetext{
${ }^{26}$ KOSKENNIEMI, M., "What Use for Sovereignty Today?", Asian Journal of International Law, vol. 1, 2011, p. 65.

${ }^{27}$ El poder de la televisión para condicionar la geopolítica se hizo muy evidente durante la evolución de la política exterior norteamericana con Bosnia Herzegovina y Kosovo. Estas guerras no amenazaban intereses clásicos de las potencias occidentales, no implicaban recursos energéticos ni inclinaban la balanza del poder global. Debido a esto EEUU y la OTAN diseñaron inicialmente una política de implicación restringida, presentando la crisis como humanitaria, no como estratégica. Con el tiempo, tras los fracasos de la UE, la OTAN y la ONU en detener la limpieza étnica en Bosnia Herzegovina, ésta se convirtió en un lugar simbólico. Las constantes imágenes televisivas e información mediática sobre masacres y genocidio minaron los fundamentos de la expansión de la OTAN en el continente. Bosnia Herzegovina se convirtió en 'estratégica' gracias a los medios, a su valor simbólico, y a su proximidad geográfica con Occidente. Con las nuevas imágenes de masacres de civiles en Kosovo en 1998 y 1999 la credibilidad de la OTAN como institución que podía mantener la paz en Europa fue de nuevo desafiada. Kosovo era, además, un potencial 'dominó étnico' cuya inestabilidad amenazaba con extenderse a pueblos y Estados vecinos. Y se convirtió, también, en 'estratégico' y su guerra humanitaria fue propulsada por la televisión como cruzada moral. La relación recíproca entre prácticas geopolíticas y medios de masas suscita preguntas críticas sobre el poder y la política global, ¿cómo y por qué algunas crisis son eventos mundiales y no otras? Kosovo se proyectó como crisis global, pero no Chechenya ni Angola, ni Timor Oriental, donde se sucedían masacres similares y la respuesta a estas cuestiones coloca en primer plano la importancia persistente no sólo de la geopolítica y la geografía continental, sino de geografías culturales y de identidad implícitas. Ó TUATHAIL, G., The Postmodern Geopolitical..., op. cit., p. 173.
} 
provisionales (PISG) y un futuro gobierno ${ }^{28}$. El Marco determina los poderes y responsabilidades de las PISG, aquéllos reservados al Representante Especial y establece una Asamblea Parlamentaria y las correspondientes ramas ejecutiva y judicial. El Representante Especial tiene poder para derogar cualquier intento de sobrepasar las competencias otorgadas, de modo que las instituciones están despojadas de la autoridad para realizar cualquier movimiento dirigido a decidir el estatus final de Kosovo ${ }^{29}$.

Una de las razones por las que las administraciones territoriales han recibido atención creciente en los últimos años, y numerosas críticas, es que el ejercicio de la autoridad política sobre territorios post conflicto parece estar en contradicción con las concepciones contemporáneas de lo que debe ser un gobierno legítimo. Mientras la legitimidad de los gobiernos nace de nociones de autodeterminación y democracia, las administraciones internacionales, mientras duran, niegan ambas cosas a las poblaciones que gobiernan. Si no se profundiza y no existen reglas y principios claros puede deducirse, desde este punto de vista, que la soberanía no tiene sentido intrínseco más allá de los objetivos a los que se supone debe servir. Resultaría lógico prescindir de ella si un ocupante se erige como fiduciario de la población y transforma el orden constitucional para perseguir el bien común, o bien si se establece una autoridad territorial enviada por Naciones Unidas o la Unión Europea, para supervisar la apropiada gestión de una región como Kosovo o Timor Oriental. Aunque las autorizaciones del Consejo de Seguridad nunca se refieren a la apropiación de la soberanía sino al mero ejercicio temporal de ciertas funciones soberanas, las implicaciones ideológicas y prácticas son enormes ${ }^{30}$. Tal y como afirma Stahn, existe una "reserva de obligaciones de gobernanza internacional" neutrales en su origen y que, por consiguiente, borran la diferencia entre soberanía y gobernanza y funcionan justificando una suerte de gobernanza cosmopolita ejercida

\footnotetext{
${ }^{28}$ Ley UNMIK 2001/09 “On a Constitutional Framework for Provisional Self-Government in Kosovo”, de 15 de mayo de 2001, disponible en www.un.org/peace/kosovo/pages/ regulations/reg01.09.html.

29 KNOLL, B. Y JOHNSTON MOLINA, K., “A Rocky Path: Kosovo's Transition to Provisional SelfGovernment", OSCE Yearbook 2002, Institute for Peace Research and Security Policy (IFSH), Hamburgo, p. 132.

${ }^{30}$ KOSKENNIEMI, M., What Use..., op. cit., p. 64.
} 
por cualquiera que esté en posición de hacerlo ${ }^{31}$. Es obvio que resulta necesario explicar cómo se organiza esta suerte de intervencionismo político y qué efectos tiene sobre los ciudadanos.

3.- La política de estándares o soberanía ganada

El Representante Especial en Kosovo Michael Steiner vinculó la decisión sobre el futuro del territorio a la idea de cumplimiento de ciertos estándares. El planteamiento no es nuevo, apareció tempranamente y con un enfoque asombrosamente particular ${ }^{32}$. Ya en noviembre de 1998, el Public International Law \& Policy Group (PILPG) propuso para la ex provincia la adopción de una estrategia de soberanía intermedia como base para estructurar la reducción del control serbio y permitir a su población ir acumulando autoridad y funciones de un modo que se mantuviesen los intereses de la población serbia minoritaria y de la comunidad internacional $^{33}$. Las claves de esta estrategia descansaban en un sistema en que el pueblo de Kosovo, a través de órganos políticos legítimos, estaría autorizado al ejercicio de ciertos derechos de soberanía mientras mantenía simultáneamente vínculos específicos con la República Federal de Yugoslavia. Como condición para la obtención de mayores niveles de soberanía, las instituciones y población de Kosovo estarían obligadas a garantizar la protección de los derechos de las minorías presentes en el territorio y respetar la integridad territorial de los estados vecinos (en

\footnotetext{
${ }^{31}$ STAHN, C., The Law and Practice..., op. cit., pp. 760 y ss.

32 Existen múltiples términos para este proceso singular: soberanía ganada, soberanía intermedia, reconocimiento escalonado, estatalidad provisional e independencia condicionada. Se ha tratado de aplicar en diversos conflictos: Palestina, Irlanda del Norte, Timor Oriental, Sudan, el Sahara occidental y Cachemira, entre otros. Véase, para profundizar en los particulares significados de tan variada terminología, WILLIAMS, P. R. y JANNOTTI PECCI, F., "Earned Sovereignty: Bridging the Gap between Sovereignty and Self-Determination", Stanford Journal of International Law, vol. 1, 2004. Para un listado de los casos concretos más relevantes donde ha sido aplicada la soberanía ganada véase HOOPER, J. R., y WILLIAMS, P. R., “Earned Sovereignty: The Political Dimension”, Denver Journal of International Law and Policy, vol. 31, marzo de 2004.

${ }^{33}$ Public International Law \& Policy Group for the International Crisis Group, Intermediate Sovereignty as a Basis for Resolving the Kosovo Crisis, 1998. El texto puede consultarse en http://www.crisisweb.org/projects/showreport.cfm?reportid=171
} 
especial Macedonia y Albania) aceptando las fronteras confirmadas por la Constitución yugoslava de 1974. Tras un periodo de tres a cinco años Kosovo podría pedir el reconocimiento internacional si así lo aprobaba la mayoría de su población en referéndum, siempre quedando sujeto por las obligaciones de proteger a las minorías, mantener las fronteras y rechazar cualquier asociación territorial o política con Albania.

Este planteamiento fue reflejado en los Acuerdos de Rambouillet y posteriormente desarrollado por un buen número de comisiones, expertos y think tanks, resultando en la formación de la doctrina de Naciones Unidas que ha dado en llamarse 'Standards before Status' (primero los estándares y después el estatus).

A través de la aplicación de esta doctrina se establecen una serie de condiciones que el candidato a soberano debe cumplir. En algunos casos, como Irak, la devolución del poder tenía cómo única condición que se celebraran elecciones y fuera elegido un gobierno. En el caso de Kosovo la lista era mucho más larga. Para formular la transferencia de competencias desde UNMIK a las nacientes instituciones provisionales de autogobierno dentro de unos parámetros de gobierno responsable, el Representante Especial Steiner inauguró una política de evaluación a través de la cual se desarrollarían indicadores en ocho áreas de gobernanza. Según el mismo Representante Especial, el fundamento de este enfoque era que Kosovo sólo podría avanzar hacia una sociedad justa si se daban determinadas condiciones previas, a la sazón, los estándares que se requerían para la potencial integración de Kosovo en las estructuras de la Unión Europea.

La hoja de ruta diseñada para Kosovo describía, de modo muy general, los logros que se consideraban necesarios pero olvidaba aportar orientaciones o directrices que ayudaran a lograrlos. En primer lugar, los estándares resultaban ser una lista de exigencias que requerían bases sociales y económicas previas, de las que Kosovo carecía tras el devastador conflicto y sin las cuales ningún Estado es viable. En segundo lugar, los estándares parecían estar encaminados explícitamente a lograr el objetivo concreto de vestir a Kosovo con los colores de Europa, permaneciendo tal vez los demás objetivos en un segundo plano ${ }^{34}$.

\footnotetext{
34 Destacando exclusivamente algunos ejemplos llamativos, mencionaremos que en el documento original donde constan los estándares a cumplir, y en el mucho más amplio documento en que figura la
} 
Incluso los autores vinculados a UNMIK se ven obligados a señalar que hasta 2003, auque la misión presentó informes regulares sobre la implementación del sistema, el proceso se desarrolló como un ejercicio exclusivo de la propia UNMIK, dada la ausencia de poder local, y era una especie de herramienta de gestión interna y no una política adoptada por las instituciones locales ${ }^{35}$. Los estándares impuestos,

estrategia diseñada posteriormente para completar los objetivos, o concretarlos al menos, se menciona a menudo la libertad de movimientos para las minorías, una vez la libertad de información y otra la de asociación en el apartado de medios de comunicación y ninguna otra libertad, tampoco se menciona ni una sola vez la protección a la infancia, por ejemplo. El primer documento, cuyo título es Standards for Kosovo (carente de ningún logo identificativo o firma y en el que no coinciden las fechas de la primera y última página), consta de 16 páginas, algunas de ellas en blanco, no fue publicado oficialmente y está disponible en http://www.unmikonline.org/standards/docs/leaflet_stand_eng.pdf. El segundo documento, titulado Kosovo Standards Implementation Plan (117 páginas) puede encontrarse en http://www.unmikonline.org/pub/misc/ksip_eng.pdf.

${ }^{35}$ Véase KNOLL, B., "From Benchmarking to Final Status? Kosovo and The Problem of an International Administration's Open-Ended Mandate", The European Journal of International Law, vol. 16, nº 4, 2005, p. 642. Pueden consultarse los siguientes documentos oficiales de Naciones Unidas sobre la aplicación de esta política: $S / 2003 / 113$ (de 29 de enero de 2003) que enfatizaba que quedaba mucho por hacer antes de cumplir los estándares; S/PV.4703 (de 6 de febrero de 2003) donde el Consejo hacía hincapié en la seguridad y el desempleo recalcando que los estándares conducentes a asegurar las necesidades de las personas debían tener preferencia sobre la determinación del estatus final de Kosovo (nótese, no sobre los estándares que no afectaban a las necesidades de los ciudadanos); S/PV. 4880 (de 12 de diciembre de 2003) expresando el Consejo su apoyo a la política de 'standards before status'; S/PRST/2003/26 (de 12 de diciembre de 2003), Declaración del Presidente del Consejo de Seguridad refrendando los "Estándares para Kosovo"; S/PV.4886 (de 17 de diciembre de 2003), sesión informativa del Vicesecretario General para Operaciones de Paz Jean-Marie Guéhenno para inaugurar la política de 'standards before status'; S/PV.4910 (de 6 de febrero de 2004), sesión informativa del Representante Especial Harri Holkeri, que enfatizaba que 'standards before status' era el núcleo del proyecto político de UNMIK; S/2004/907 (de 17 de noviembre de 2004), se hace eco de que el progreso hacia los ocho estándares era 'limitado e irregular'; S/PRST/2004/13 (de 30 de abril de 2004), reiterando el apoyo firme a la política de 'standards before status' e instando a las PISG a centrar sus esfuerzos en dos estándares clave: retorno de refugiados y protección de las minorías y libertad de movimientos; S/2005/88 (de 14 de febrero de 2005) hace notar un progreso tangible hacia el cumplimiento de los estándares pero considerando que ninguno había sido logrado por completo; S/2005/335 y Corr. 1 (de 26 de mayo de 2005), incluye un anexo destacando los logros hasta el momento y los retos pendientes en materia de instituciones democráticas, imperio de la ley, libertad de movimientos, retorno de refugiados y derechos de las minorías, economía, derechos de propiedad, patrimonio cultural, diálogo y KPC; S/PV.5188 (de 27 de mayo de 2005), sesión informativa del Representante Especial Søren Jessen-Petersen enfatizando los logros en los estándares pero destacando la todavía baja participación de las minorías; S/2005/635 (de 7 de octubre de 2005), carta del Secretario General al Presidente del Consejo de Seguridad transmitiendo el Informe Eide que destacaba los logros hasta la fecha y apoyaba el comienzo de conversaciones sobre el estatus; S/PRST/2005/51 (de 24 de octubre de 2005) donde se declaraba que era el momento de comenzar el proceso político para determinar el estatus de Kosovo y se urgía a continuar el compromiso 
a través de los cuales Kosovo debía probar que estaba preparado para el inicio del debate sobre su estatus y en cuya implementación los progresos deberían ser 'cuantificables' eran los siguientes:

1. - Funcionamiento de Instituciones democráticas representativas y efectivas. Los objetivos incluían consolidación de la participación política, acceso a los servicios públicos y al funcionariado de las minorías étnicas, recaudación de impuestos de rentas, provisión eficiente de servicios públicos y medios de comunicación independientes.

2. - Imperio de la Ley. Incluye desmantelamiento de redes criminales, fin de la violencia extremista, respeto público por la policía y el poder judicial, imparcialidad de los jueces y los cuerpos de la policía (KPS), procesamiento de criminales y garantías de juicio justo para cualquier acusado, asimismo representación suficiente de las minorías.

3. - Libertad de movimientos. Todas las comunidades étnicas deben poder circular libremente y usar su lengua, sin intervención del ejército o la policía, por el territorio de Kosovo.

4. - "Retorno sostenible” de refugiados y derechos de las minorías étnicas. Respeto al derecho de todas las comunidades étnicas de permanecer en Kosovo, garantía de su derecho a mantener sus propiedades y a retornar a ellas.

5. - Economía. Los objetivos incluyen la creación de una sólida base institucional para la economía de mercado y un sistema mejorado de recaudación de impuestos y tasas, un presupuesto equilibrado y la privatización de bienes de propiedad social.

6. - Derechos de Propiedad. Toda la propiedad, incluyendo residencias, tierras, empresas y bienes sociales deberá tener un titular definido y legítimo.

7. - Diálogo con Belgrado. Kosovo deberá tener relaciones normales con la República Federal de Yugoslavia y con los demás países vecinos, lo que incluye contacto directo de las PISG con sus homólogos.

de la implementación de estándares; S/2006/45 (de 25 de enero de 2006) haciendo notar que el progreso en la implementación de estándares se había ralentizado con respecto a periodos previos. 
8. - Kosovo Protection Corps. La institución debe reducirse considerablemente y debe incluir activamente a las minorías étnicas presentes en el territorio ${ }^{36}$.

Esta política de 'standards before status' se desarrolla en el contexto de aquello que algunos teóricos denominan soberanía ganada ${ }^{37}$. Entra en juego lo que hemos descrito como gradación de la soberanía, aunque lo que las instituciones de Kosovo van adquiriendo son pequeñas parcelas de gobernanza, y en absoluto poderes soberanos de ningún tipo. Imaginamos que la gradación está presente de algún modo, por ejemplo, uno puede suponer que si Kosovo cumple uno de los estándares está un grado más cerca de la decisión sobre su soberanía. Pero en todos los años de mandato UNMIK ninguna institución kosovar logró estar en la situación de decidir sin que haya una instancia internacional superior que pueda rectificar o anular la decisión si lo desea o lo considera oportuno. Se hace imprescindible, además, aclarar que esta política implantada en Kosovo no estaba encaminada a ganar ninguna soberanía, no se lograría el estatus de Estado en caso de cumplir todos los estándares, estas condiciones sólo abrían la puerta a iniciar conversaciones sobre el futuro de Kosovo, que pudiera resultar en verse de nuevo bajo la soberanía serbia. En cualquier caso, no nos parece del todo acertado utilizar el término de "soberanía suspendida" para el caso de Kosovo ${ }^{38}$, dado que las competencias y funciones soberanas se ejercen efectivamente en todo momento, no están suspendidas a la espera de una decisión final, están sencillamente depositadas en un

\footnotetext{
${ }^{36}$ Resulta muy llamativo que la sin duda necesaria reestructuración del KPC esté sin embargo al mismo nivel que el imperio de la ley o la democracia efectiva.

${ }^{37}$ KNOLL, B., From Benchmarking..., op. cit., p. 640. No debe confundirse la soberanía ganada con la soberanía compartida, que se refiere a la inclusión de actores internacionales en instituciones locales de Estados emergentes. Este tipo de soberanía no divide el poder entre instituciones, ni supone una cooperación entre actores soberanos, ni amenaza el marco normativo de la soberanía, describe simplemente la integración de expertos externos en las estructuras de gobernanza de un Estado naciente. Véase al respecto KRASNER, S., "Sharing Sovereignty: New Institutions for Collapsed and Failing States", International Security, vol. 29, no 2, 2004.

38 YANNIS, A., "The Concept of Suspended Sovereignty in International Law and its Implications in International Politics", European Journal of International Law (2002), vol. 13, no 5, pp. 1037-1052.
} 
actor no estatal ${ }^{39}$. Es decir que, a pesar de que Kosovo no se autogobierna, es indudablemente gobernado.

Según sus defensores, la "soberanía ganada", como herramienta de resolución de conflictos, se ha desarrollado como un proceso flexible que se implementa a través de un periodo de tiempo variable y está definida por tres elementos esenciales: la soberanía compartida, la construcción de instituciones y la determinación del estatus final. El proceso puede también llevar aparejados tres elementos opcionales: soberanía en fases, soberanía condicionada y soberanía constreñida. Estos elementos sirven para adaptar el enfoque de la soberanía ganada a las circunstancias únicas de cada conflicto y las necesidades particulares de las partes $^{40}$.

Todas estas variaciones han tenido lugar en el contexto de las administraciones internacionales, en el Sahara Occidental (Plan de Paz) y Timor Oriental el acceso a la independencia estaba vinculado a una transferencia gradual de autoridad por parte de una entidad gobernante (Marruecos y la misión UNTAET respectivamente) a un pueblo tras la materialización de una opción sobre el estatus. Kosovo es un caso claro de soberanía condicionada y Bosnia Herzegovina e Irak constituyen ejemplos de soberanía limitada en su ejercicio por una presencia internacional continuada, ya sea esta administrativa, militar, o ambas cosas ${ }^{41}$.

A la vista de estas consideraciones parece necesario ampliar la visión del principio de soberanía y poder contemplarlo como un espectro, reconociendo una gama de estatus soberanos intermedios que forman parte de este. La soberanía intermedia y los conceptos a ella asociados, conceptos como independencia condicionada, estatalidad provisional o soberanía diferida, pueden tener la forma de mayor autonomía para una comunidad, reconocimiento ganado o reconocimiento

\footnotetext{
${ }^{39}$ Sólo la soberanía que implica establecimiento de fronteras, aquélla necesaria para decidir el estatus final parece no poder ser ejercida por ninguno de los actores presentes.

${ }^{40}$ WILLIAMS, P. R. y JANNOTTI PECCI, F., Earned Sovereignty..., op. cit., p. 4.

${ }^{41}$ STAHN, C., The Law and Practice..., op. cit., p. 757.
} 
escalonado. El término pretende, según parece, describir una entidad que es algo menos que un Estado soberano, pero más que una entidad sub-estatal ${ }^{42}$.

La soberanía ganada, además de los problemas y confusiones teóricas que lleva consigo y aquellos relacionados con la legalidad y la legitimidad de las políticas de condicionalidad, entraña ciertos peligros nada desdeñables. En Kosovo resultó en la retención del poder por parte de UNMIK por un periodo tan prolongado que obligaba a que fuese la misión la única responsable de cumplir los estándares y el único elemento al que culpar de potenciales fallos. La renuencia de los actores internacionales en Kosovo a transferir la autoridad atascó el proceso y desacreditó por completo el planteamiento, que, por otra parte dejaba abierta la cuestión de cómo iba a tener lugar la decisión sobre el estatus final del territorio kosovar. Como consecuencia, la condicionalidad sobre la que descansaba la soberanía ganada ofrecía nulos incentivos a quienes debían someterse a ella. Resulta todavía más preocupante el hecho de que las instituciones de Kosovo debían cumplir unos objetivos, de los cuales un buen número se encontraba completamente fuera de su control: el diálogo con Serbia no resultaba posible si Belgrado no deseaba dialogar, la privatización y liquidación de bienes sociales fue una de las 'competencias reservadas' que UNMIK se atribuyó desde el primer momento, y así sucesivamente.

La política de estándares o soberanía ganada ha supuesto para UNMIK y por ende para la comunidad internacional, un rotundo fracaso, y para Kosovo una importante fuente de perjuicios. El 'Informe Eide', remitido al Secretario General de la ONU a mediados de 2004, recomendaba la sustitución inmediata de esta estrategia por un enfoque más dinámico ${ }^{43}$. En junio de 2005 se nombró un Enviado Especial (el embajador Eide, autor del informe), encargado de conducir la revisión de los estándares y examinar las condiciones para poner en marcha un nuevo proceso de avance. El Parlamento Europeo, en una sesión especial, también se pronunció

\footnotetext{
${ }^{42}$ SCHARF, M. P., Earned Sovereignty: Juridical..., op. cit., p. 375.

${ }^{43}$ The Situation in Kosovo. Report to the Secretary General of the United Nations. Summary and Recommendations, escrito por el embajador Kai Eide, director de la Misión Política para Kosovo de la DPKO, S/2004/932, Annexo I, Bruselas, 15 de julio de 2004.
} 
diciendo que la política de Naciones Unidas 'standards before status' en Kosovo no había dado los resultados esperados tras cinco años y que debía ser abandonada ${ }^{44}$.

Esta especie de redefinición de la soberanía, central para el marco de la construcción del Estado por parte de Naciones Unidas o statebuilding, facilita la erosión de los lazos entre el poder y la responsabilidad, permitiendo a los actores internacionales distanciarse de las políticas que ellos mismos promueven. Además, como se ha visto, su eficacia es, cuanto menos, dudosa. Los Estados sin capacidad verdadera de autogobierno permanecen débiles y sus autoridades carecen de legitimidad. La agenda política se centra en mecanismos burocráticos y administrativos, capaces únicamente de institucionalizar las actuales divisiones entre Occidente y no-Occidente, inútiles para hacerse cargo de las divisiones sociales y políticas de un Estado que resurge de un conflicto ${ }^{45}$.

La soberanía sigue siendo crucial, tanto para la comunidad internacional como para el pueblo de Kosovo, que la considera esencial para el funcionamiento del Estado como componente de pleno derecho de la comunidad internacional. El hecho de hacer depender esta soberanía del logro de unos estándares impuestos desde el exterior hace que pierda su otrora lugar principal en la escala de importancia de la agenda internacional. La ausencia de la sociedad en el ejercicio de la construcción del Estado ha generado resultados inorgánicos y una estatalidad sin significación política. La presencia internacional en Kosovo permanece fuerte durante años en los tres poderes del Estado, a pesar de que la promesa vacía de resolución del estatus final es periódicamente renovada. Esto no puede sino frustrar la naturaleza verdadera de la estatalidad en Kosovo.

\section{4.- Conclusiones}

El derecho global de hoy es anti-formalista y se nos dice que "lo que cuenta es la existencia de un poder de facto, cualquiera que sea su origen y objetivos. Sólo ese

\footnotetext{
${ }^{44}$ Parlamento Europeo, Kosovo: 'standards and status' should go hand in hand, Bruselas, 26 de enero de 2005, en http://www.europa-eu-un.org/articles/en/article_4265_en.htm.

45 CHANDLER, D., "International State-Building: Beyond Conditionality, Beyond Sovereignty", Guest Seminar, Royal Institute for International Relations (IRRI-KIIB), Bruselas, 17 de noviembre de 2005, p. 4.
} 
poder alcanzará el bien común para todos"46. Esta aseveración no es una extravagancia postmoderna, la soberanía no apareció como invención filosófica sino que fue producto del cansancio de Europa por los conflictos religiosos y su propósito era práctico: acabar con el poder papal e imperial y pacificar las sociedades europeas. La clave estaba precisamente en apartarse de lo trascendental y acercarse al poder del productor de normas secular que estaría controlado para lograr el beneficio de la población. Sin poder, ni la seguridad ni el bienestar pueden existir y el vínculo entre protección y obediencia queda roto, permitiendo que emerja la anarquía $^{47}$.

La globalización ha supuesto la autoridad creciente de los lenguajes técnicos y científicos, cuyos hablantes nativos están localizados casi exclusivamente en Occidente y para los cuales los seres humanos aparecen como objetos de "protección" o caridad pero raramente como dueños de sus propias vidas. Propone nuevos tipos de valor y preferencias que acompañan la aparición de nuevas clases profesionales y se presentan en términos neutrales, científicos y no políticos. Lo que se necesita del derecho internacional es la politización de este proceso, la creación de plataformas y vocabularios que subrayen la naturaleza contestable de la globalización. El derecho internacional puede hacer esto sólo si, en vez de convertirse en otro vocabulario técnico de la gobernanza global, buscara ser el foro en que se toman decisiones globales de forma transparente y promoviera la responsabilidad de las clases profesionales ante las comunidades afectadas por sus discutibles preferencias. El derecho internacional no tiene respuestas rígidas sobre cómo el mundo debe ser gobernado. Pero puede ser usado como vocabulario para articular preferencias alternativas y llevar a cabo maniobras para limitar los poderes de las clases ejecutivas globales y grupos de expertos. Esto significaría, inevitablemente, resaltar la importancia del vocabulario de la soberanía política como expresión de preferencias y valores locales así como de tradiciones de

\footnotetext{
${ }^{46}$ KOSKENNIEMI, M., What Use..., op. cit., p. 68.

47 Véase KOSKENNIEMI, M., From Apology to Utopia. The Structure of International Legal Argument, Cambridge University Press, 2005, p. 17. Véase también FERRAJOLI, L., "La crisis de la democracia en la Era de la Globalización”, Anales de la Cátedra Francisco Suárez, IVR 2005, pp. 37-51.
} 
autogobierno, autonomía, y debate político constante. En una palabra, debería contribuir a re-imaginar lo que la política internacional significa hoy ${ }^{48}$.

Las aproximaciones jurídicas a las situaciones de conflicto interno, violación masiva de los derechos y Estados fallidos están lejos de obtener un consenso y causan tensión política a nivel global y, a menudo, bloqueo en los procesos e intentos de solucionar o atenuar los conflictos. A esto se añade la práctica inexistencia de estas aproximaciones para aquéllos casos en que la soberanía de ciertos Estados parece abarcar el derecho a dominar a otros más débiles o vulnerar los derechos de ciudadanos no "nuestros", tanto fuera como dentro de sus fronteras. Nos encontramos, de este modo, ante un número indefinido y creciente de situaciones excepcionales cuya solución es imprevisible. No se puede seguir defendiendo la separación entre el derecho internacional y la política en ningún foro.

No encarar los complejos problemas del intervencionismo post bellum, entre ellos la frontera entre soberanía y gobernanza, su legitimación y la responsabilidad política exigible, en la sociedad mundial en que nos encontramos, causa perjuicios a

\footnotetext{
${ }^{48}$ Para evitar que el derecho internacional pierda su independencia respecto a la política internacional, el pensamiento jurídico debe batallar en dos frentes. Por un lado, debe asegurar la normatividad del derecho creando una distancia entre él y los comportamientos estatales, su voluntad y sus intereses. Por otro lado, debe asegurar la concreción del derecho distanciándolo de la moral natural. Un derecho que se solape con el comportamiento, voluntad e intereses del Estado es equivalente a una disculpa no normativa, una mera descripción social. Un derecho que se base en principios sin relación con el comportamiento, voluntad e intereses del Estado parecería utópico, incapaz de demostrar su propio contenido de un modo fiable. Para demostrar que existe el derecho internacional, con algún grado de realidad, el jurista moderno debe demostrar que el derecho es, simultáneamente, normativo y concreto y vincula al Estado independientemente de su comportamiento, deseos o intereses a la vez que puede ser verificado por referencia a dicho comportamiento, deseos e intereses. No es difícil ver que existe un peligro constante de que el derecho se diluya en una disculpa política, no puede extrañarnos dado que se entiende que el derecho existe para lograr unos fines sociales y los desacuerdos respecto a los fines correctos están a la orden del día. Esta afirmación es particularmente cierta respecto al derecho internacional, que, al igual que la política internacional, emerge de los deseos e intereses subjetivos y políticamente motivados de los Estados. La creación del derecho internacional surge de la política y la diplomacia pero debe ser separable de éstas, debe ser vinculante a pesar de los intereses del Estado contra el que se invoque, o tendremos que aceptar la exclusiva naturaleza política del derecho. Si por otra parte el derecho se mantiene por completo independiente de la práctica de los Estados individuales se tornaría un código normativo cerrado que preexistiría a los intereses de éstos, como cuando se entendía que el derecho tenía existencia autónoma como designio divino o propósito natural. La doctrina moderna, carente de la fe necesaria para esta asunción, entiende el derecho como creación artificial, basada en el comportamiento y los intereses de los Estados. Véase KOSKENNIEMI, M., What Use..., op. cit., p. 68.
} 
millones de ciudadanos. No deja de ser arriesgado, pues, mirar hacia otro lado mientras se transforma el modelo de Estado nacional de la modernidad, y resulta negligente pensar en una especie de determinismo soberano, que nos exime de la necesidad permanente de argumentar y profundizar en la exigencia de garantías para los derechos de modo universal. El derecho no puede seguir sucumbiendo a la fuerza ciertamente pero tampoco debe renunciar a la lucha por un orden jurídico internacional racionalizado y socialmente legitimado

“(y) si es verdad que en tiempo breve no podemos hacernos ilusiones, también es verdad que la historia nos enseña que los derechos no caen del cielo y un sistema de efectivas garantías no nace en la discusión académica, ni se construye en pocos años y tampoco en pocas décadas. Como sabemos, es el fruto de largos y fatigosos procesos, de choques y de conflictos. Así ha sido para el Estado de derecho (...) sería absurdo pensar que no será así para el derecho internacional y también lo sería no comprometernos (...) en la batalla por la elaboración y por la afirmación de esas garantías" ${ }^{49}$.

La crítica jurídica del derecho y de las situaciones existentes exige no sólo la denuncia de su ilegalidad sino también de su injusticia. Aunque sólo se trate de aproximarse al cumplimiento de algunas de estas ideas, la exigencia de cambio en las soluciones aportadas por el derecho resulta ineludible. Como apuntaba Ferrajoli, "no podemos reclamar al Derecho lo que no puede dar. Pero tampoco hemos de renunciar a pedirle lo que puede dar" ${ }^{, 50}$.

\footnotetext{
49 FERRAJOLI, L., "La Conquista de América y la Doctrina de la Soberanía Exterior de los Estados", en BERGALLI, R., y RESTA, E., Soberanía: Un Principio que se Derrumba, Paidós, Barcelona, 1996, p. 176.

50 FERRAJOLI, L., "Estado de Derecho: entre Pasado y Futuro", Cambio de paradigma en la Filosofía Política, Fundación Juan March, Madrid, 2001, p. 136.
} 